University of Nebraska - Lincoln

DigitalCommons@University of Nebraska - Lincoln

July 1988

\title{
Variational Calculation of Multiphoton Ionization Processes for the $\mathrm{H}$ Atom
}

Bo Gao

University of Nebraska - Lincoln

Anthony F. Starace

University of Nebraska-Lincoln, astarace1@unl.edu

Follow this and additional works at: https://digitalcommons.unl.edu/physicsstarace

Part of the Physics Commons

Gao, Bo and Starace, Anthony F., "Variational Calculation of Multiphoton Ionization Processes for the $\mathrm{H}$ Atom" (1988). Anthony F. Starace Publications. 35.

https://digitalcommons.unl.edu/physicsstarace/35

This Article is brought to you for free and open access by the Research Papers in Physics and Astronomy at DigitalCommons@University of Nebraska - Lincoln. It has been accepted for inclusion in Anthony F. Starace Publications by an authorized administrator of DigitalCommons@University of Nebraska - Lincoln. 


\title{
Variational Calculation of Multiphoton Ionization Processes for the $\mathbf{H}$ Atom
}

\author{
Bo Gao and Anthony F. Starace \\ Department of Physics and Astronomy, The University of Nebraska, Lincoln, Nebraska 68588
}

(Received 14 April 1988)

\begin{abstract}
Well-known variational principles for second-order perturbation matrix elements are extended to higher-order multiphoton processes for atomic hydrogen. Numerical applications to two- and threephoton ionization of the hydrogen ground state provide results comparable to those of analytic calculations, even near intermediate-state resonances, yet without the complications involved in summation over intermediate states. Extensions to multiphoton processes for many-electron atoms are discussed briefly.
\end{abstract}

PACS numbers: $32.80 . \mathrm{Rm}, 31.15 .+\mathrm{q}$

In calculating $N$ th-order perturbation matrix elements $(N \geq 2)$, such as in higher-than-first-order Born approximations, multiphoton processes, or higher-than-first-order correlation effects, one always encounters the problem of summing over a complete set of intermediate states. Use of the Dalgarno-Lewis procedure ${ }^{1}$ reduces the problem formally to the solution of a set of $N-1$ coupled inhomogeneous differential equations. However, the accuracy of the numerical solution of these equations becomes an increasing concern as $N$ becomes large. While the use of variational principles to improve this accuracy has been common for the calculation of many second-order processes, ${ }^{2}$ such as, e.g., frequencydependent dipole polarizabilities ${ }^{3,4}$ or, more generally, matrix elements of the Green's function, ${ }^{5}$ their use for higher-than-second-order processes (and for multiphoton processes in particular ${ }^{6}$ ) has been rare.

Consider more specifically the case of multiphoton processes, for which the numerical evaluation of the $N$ th-order transition amplitude is the key problem in theoretical calculations. For the special case of atomic hydrogen, numerical methods ${ }^{7-10}$ based on the Dalgarno-Lewis procedure ${ }^{1}$ have been complemented by a number of analytic approaches. Some of these evaluate the second-order perturbative matrix element analytical-

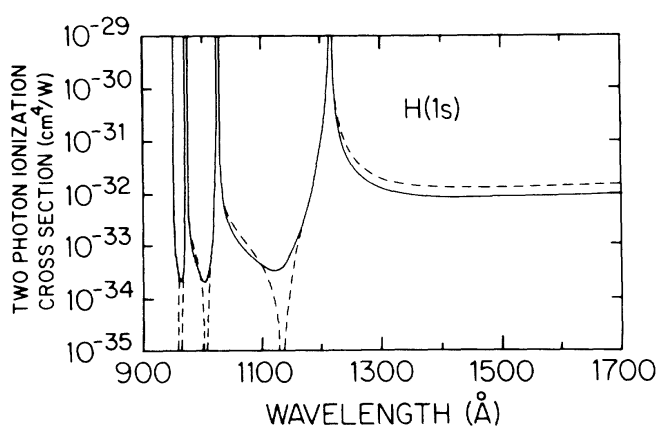

FIG. 1. Two-photon ionization cross section $\left(\mathrm{cm}^{4} / \mathrm{W}\right)$ of $\mathrm{H}(1 s)$ vs photon wavelengths $(\AA)$ for photon energies below the one-photon ionization threshold. Dashed curve, circular polarization. Solid curve, linear polarization. ly. ${ }^{11-13}$ Others ${ }^{14-18}$ employ analytic expansions of the Coulomb Green's function, which permit the evaluation of higher-order perturbation matrix elements. The numerical difficulties of the evaluation of multiphoton transition amplitudes by these methods are well known. $9,15,17,18$ Furthermore, the often significant differences between the predictions of even the analytic calculations for atomic hydrogen do not augur well for accurate theoretical calculations of higher-order processes for nonhydrogenic systems, although significant recent theoretical progress has been made for alkali-metal atoms ${ }^{10,19,20}$ and for rare-gas atoms. ${ }^{21,22}$

We present here a variational principle for the $N$ thorder multiphoton transition matrix element for a hydrogenic system which addresses the major difficulty in evaluating higher-order amplitudes, namely, the summation over infinite numbers of intermediate states. Our expression for such amplitudes is variationally stationary with respect to changes in the approximations to these summations. Numerical applications of this variational method to the two- and three-photon ionization cross sections for $\mathrm{H}(1 s)$ are presented and compared with the analytic predictions of others. Extensions of the variational principle to nonhydrogen systems for a number of atomic processes are being developed, ${ }^{23}$ but are only dis-

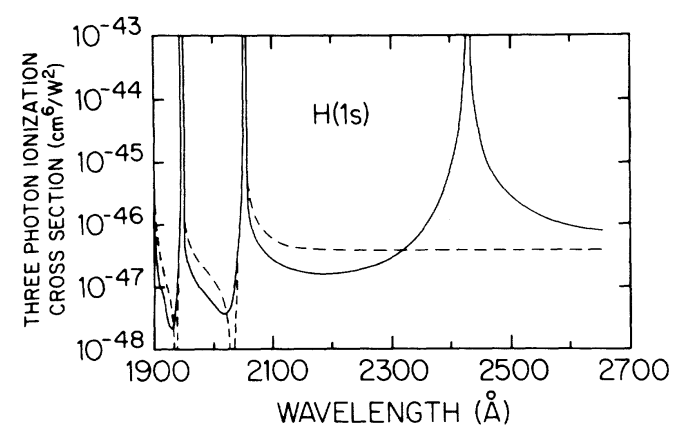

FIG. 2. Three-photon ionization cross section $\left(\mathrm{cm}^{6} / \mathrm{W}^{2}\right)$ of $H(1 s)$ vs photon wavelengths $(\AA)$ for photon energies below the two-photon ionization threshold. Dashed curve, circular polarization. Solid curve, linear polarization. 
TABLE I. Two-photon ionization cross section $\left(\mathrm{cm}^{4} / \mathrm{W}\right)$ of $\mathrm{H}(1 s)$ for linearly polarized light below the one-photon ionization threshold.

\begin{tabular}{|c|c|c|c|c|c|c|c|c|}
\hline$\lambda(\AA)$ & $\mathrm{CT}^{\mathrm{a}}$ & $\mathrm{K}^{\mathrm{b}}$ & $\mathrm{GT}^{\mathrm{c}}$ & LDFJR $^{d}$ & $K V^{e}$ & $\mathrm{CP}^{\mathrm{f}}$ & $\mathrm{Ka}^{\mathrm{g}}$ & Present \\
\hline 975 & $5.152 \times 10^{-33}$ & & & $4.923 \times 10^{-33}$ & $6.773 \times 10^{-33}$ & & $4.91 \times 10^{-33}$ & $4.975 \times 10^{-33}$ \\
\hline 1020 & $6.752 \times 10^{-33}$ & & $5.522 \times 10^{-33}$ & $7.080 \times 10^{-33}$ & $5.522 \times 10^{-33}$ & $7.08 \times 10^{-33}$ & $7.09 \times 10^{-33}$ & $7.154 \times 10^{-33}$ \\
\hline 1100 & $4.013 \times 10^{-34}$ & $4.049 \times 10^{-33}$ & $4.049 \times 10^{-33}$ & $4.035 \times 10^{-34}$ & $4.049 \times 10^{-34}$ & & $4.00 \times 10^{-33}$ & $4.024 \times 10^{-34}$ \\
\hline 1200 & $6.303 \times 10^{-32}$ & $5.803 \times 10^{-32}$ & $5.803 \times 10^{-32}$ & $6.417 \times 10^{-32}$ & $5.803 \times 10^{-32}$ & $6.42 \times 10^{-32}$ & $6.42 \times 10^{-32}$ & $6.441 \times 10^{-32}$ \\
\hline 1300 & $1.276 \times 10^{-32}$ & $1.283 \times 10^{-32}$ & $1.283 \times 10^{-32}$ & $1.277 \times 10^{-32}$ & $1.283 \times 10^{-32}$ & & $1.27 \times 10^{-32}$ & $1.276 \times 10^{-32}$ \\
\hline 1400 & $8.450 \times 10^{-33}$ & $8.453 \times 10^{-33}$ & $8.453 \times 10^{-33}$ & $8.470 \times 10^{-33}$ & $8.453 \times 10^{-33}$ & & $8.45 \times 10^{-33}$ & $8.451 \times 10^{-33}$ \\
\hline 1600 & $9.154 \times 10^{-33}$ & $9.143 \times 10^{-33}$ & $9.143 \times 10^{-33}$ & $9.182 \times 10^{-33}$ & $9.143 \times 10^{-33}$ & & $9.15 \times 10^{-33}$ & $9.153 \times 10^{-33}$ \\
\hline 1700 & $1.025 \times 10^{-32}$ & $1.024 \times 10^{-32}$ & $1.023 \times 10^{-32}$ & $1.028 \times 10^{-32}$ & $1.024 \times 10^{-32}$ & & $1.03 \times 10^{-32}$ & $1.025 \times 10^{-32}$ \\
\hline
\end{tabular}

${ }^{a}$ Chan and Tang, Ref. 12

${ }^{\mathrm{b}}$ Klarsfeld, Ref. 13b.

${ }^{\mathrm{c}}$ Gontier and Trahin, Ref. 8.

${ }^{\mathrm{d}}$ Laplanche et al., Ref. 15.
${ }^{\mathrm{e}}$ Khristenko and Vetchinkin, Ref. 16

${ }^{f}$ Chang and Poe, Ref. 9.

${ }^{8}$ Karule, Ref. 14b.

cussed briefly here.

In lowest-order perturbation theory, with use of the electric-dipole approximation, the ionization cross section for transitions from the initial hydrogenic (bound) state $|n l\rangle$ to the final hydrogenic (continuum) state $|E L\rangle$ by $N$ polarized photons may be expressed in terms of the following radial matrix elements ${ }^{7}$ :

$$
\begin{aligned}
P\left(l_{1}, l_{2}, \ldots, l_{N-1}, L \mid \hbar \omega\right)=\langle E L| r\left[E_{n}+\right. & \left.(N-1) \hbar \omega-h\left(l_{N-1}\right)\right]^{-1} \\
& \times r\left[E_{n}+(N-2) \hbar \omega-h\left(l_{N-2}\right)\right]^{-1} r \cdots r\left[E_{n}+\hbar \omega-h\left(l_{1}\right)\right]^{-1} r|n l\rangle .
\end{aligned}
$$

Equation (1) hides the $N-1$ summations over intermediate states by repeated use of the closure relation. ${ }^{24}$ In Eq. (1), $|n l\rangle$ represents the radial wave function and $E_{n}$ represents the energy for the bound state having principal quantum number $n$ and orbital angular momentum $l ;\langle E L|$ represents the radial wave function for the continuum final state with electron energy $E$ and orbital angular momentum $L ; l_{1}, l_{2}, \ldots, l_{N-1}$ represent one particular set of intermediate-state orbital angular momenta- the total $N$-photon ionization cross section is expressed in terms of a summation of the square of the absolute value of the amplitude in Eq. (1) over different sets of $l_{i}{ }^{7}{ }^{7}$; finally, the one-electron radial Hamiltonian for a particular orbital angular momentum $l_{i}$ is defined by

$$
h\left(l_{i}\right) \equiv-\frac{1}{2} d^{2} / d r^{2}-1 / r+l_{i}\left(l_{i}+1\right) / 2 r^{2} .
$$

Define now the following states:

$$
\begin{aligned}
& |\lambda\rangle \equiv\left[E_{n}+(N-1) \hbar \omega-h\left(l_{N-1}\right)\right]^{-1} r\left[E_{n}+(N-2) \hbar \omega-h\left(l_{N-2}\right)\right]^{-1} r \cdots r\left[E_{n}+\hbar \omega-h\left(l_{1}\right)\right]^{-1} r|n l\rangle, \\
& \left\langle\lambda^{\prime}\right| \equiv\langle E L| r\left[E_{n}+(N-1) \hbar \omega-h\left(l_{N-1}\right)\right]^{-1} r \cdots r\left[E_{n}+\hbar \omega-h\left(l_{1}\right)\right]^{-1},
\end{aligned}
$$

where $|\lambda\rangle\left(\left\langle\lambda^{\prime}\right|\right)$ is the state resulting from $(N-1)$-fold excitation (deexcitation) of $|n l\rangle(\langle E L|)$. In terms of the states $|\lambda\rangle$ and $\left\langle\lambda^{\prime}\right|$, the radial matrix elements in Eq. (1) may be written in three different ways, which may be combined to form the following expression:

$$
\begin{aligned}
& P\left(l_{1}, l_{2}, \ldots, l_{N-1}, L \mid \hbar \omega\right)=\langle E L|r| \lambda\rangle+\left\langle\lambda^{\prime}|r| n l\right\rangle \\
& \quad-\left\langle\lambda^{\prime}\right|\left[E_{n}+\hbar \omega-h\left(l_{1}\right)\right] r^{-1}\left[E_{n}+2 \hbar \omega-h\left(l_{2}\right)\right] r^{-1} \\
& \cdots r^{-1}\left[E_{n}+(N-1) \hbar \omega-h\left(l_{N-1}\right)\right]|\lambda\rangle,
\end{aligned}
$$

which is easily seen to be variationally stationary with respect to changes in $|\lambda\rangle$ and $\left\langle\lambda^{\prime}\right|$. For two-photon processes, Eq. (4) reduces to the form of the variational principle developed for the $T$-matrix element for scattering processes by Nuttall and Cohen. ${ }^{25}$ In our numerical calculations we represent the states $|\lambda\rangle$ and $\left\langle\lambda^{\prime}\right|$ as linear combinations of $M$ Slater functions of the form $r^{\left(l_{N-1}+i\right)} e^{-\beta r}$ and $r^{\left(l_{1}+i\right)} e^{-\beta r}$, respectively, where $1 \leq i \leq M$. In addition, the function $r^{N-1}|n l\rangle$ is included in $|\lambda\rangle$ and $r^{N-1}|E L\rangle$ is included in $\left|\lambda^{\prime}\right\rangle$ to speed convergence; they represent the dominating terms in the asymptotic forms of $|\lambda\rangle$ and $\left|\lambda^{\prime}\right\rangle$. The matrix elements in Eq. (4) are evaluated analytically and the coefficients of the Slater functions are evaluated by the requirement that Eq. (4) be variationally stationary. 
TABLE II. Two-photon ionization cross section $\left(\mathrm{cm}^{4} / \mathrm{W}\right)$ of $\mathrm{H}(1 s)$ for linearly polarized light above the one-photon ionization threshold.

\begin{tabular}{cccc}
\hline \hline$\lambda(\AA)$ & $\mathrm{Kl}^{\mathrm{a}}$ & $\mathrm{Ka}^{\mathrm{b}}$ & Present \\
\hline 200 & $\ldots$ & $3.02 \times 10^{-38}$ & $2.990 \times 10^{-38}$ \\
400 & $\cdots$ & $2.15 \times 10^{-36}$ & $2.156 \times 10^{-36}$ \\
600 & $2.611 \times 10^{-35}$ & $2.62 \times 10^{-35}$ & $2.619 \times 10^{-35}$ \\
800 & $1.572 \times 10^{-34}$ & $1.58 \times 10^{-34}$ & $1.577 \times 10^{-34}$ \\
\hline \hline
\end{tabular}

${ }^{\mathrm{a}}$ Klarsfeld, Ref. $13 \mathrm{~b}$.

${ }^{b}$ Karule, Ref. 14b.

For two- and three-photon ionization of $\mathrm{H}(1 s)$ below the one- and two-photon ionization thresholds respectively, we used $\beta=0.5$ and $M=40$. Our results converged to ten or more significant digits for all photon frequencies, even those quite close to intermediate-state resonances, with the exception of frequencies approaching the oneand two-photon ionization thresholds, respectively. For photon frequencies above the one-photon ionization threshold in the case of two-photon ionization of $\mathrm{H}(1 s)$, we find that more care in choosing the value of $\beta$ is necessary to obtain adequate convergence. In particular, $\beta$ must be chosen to be a complex number. ${ }^{26}$ In this case, with $M=40$, convergence is only obtained to four significant figures, although we are still investigating improved numerical procedures for the case of abovethreshold ionization.

Our variational results for the two- and three-photon ionization cross sections of $\mathrm{H}(1 s)$ below the one- and two-photon ionization thresholds respectively, are shown in Figs. 1 and 2. Numerical values at selected wavelengths are compared with the results of others in Tables I, II, and III. In the two-photon ionization case, our results in Table I lie within 1\% of those of all others for wavelengths above $1200 \AA$; for wavelengths of $1200 \AA$ and smaller, our results in Tables I and II agree best with those of Laplanche et al. ${ }^{15}$ and Karule. ${ }^{14 b}$ In the three-photon ionization case, our results in Table III agree best with those of Laplanche et al. ${ }^{15}$
Generalization of the variationally stable amplitude in Eq. (4) to multielectron atoms is complicated because of the difficulty of separating angular and radial factors. An exception is the special case of two-photon processes, for which it is not necessary to compute the inverse of the dipole operator. For higher-order multiphoton processes, Eq. (4) may be cast in the form of a perturbation expansion in the electron correlation operator. Generalization of Eq. (4) for high-order processes mediated by operators other than the dipole operator is straightforward. Our work on these topics will be presented elsewhere. $^{23}$

We thank L. Spruch for helpful discussions and correspondence. This work was supported in part by $\mathrm{Na}$ tional Science Foundation Grant No. PHY-8601429.

\footnotetext{
${ }^{1}$ A. Dalgarno and J. T. Lewis, Proc. Roy. Soc. London A 233, 70 (1955).

${ }^{2}$ E. Gerjuoy, A. R. P. Rau, and L. Spruch, Rev. Mod. Phys. 55, 725 (1983)

${ }^{3}$ C. Schwartz, Ann. Phys. (N.Y.) 6, 156, 170 (1959).

${ }^{4}$ Y. M. Chan and A. Dalgarno, Proc. Phys. Soc. London 85, 227 (1965).

${ }^{5}$ T. N. Rescigno and C. W. McCurdy, Phys. Rev. A 31, 624 (1985).

${ }^{6}$ G. A. Victor, Proc. Phys. Soc. London 91, 825 (1967). Note that while wave functions were calculated variationally in this paper, the $T$-matrix amplitudes were not.

${ }^{7}$ Y. Gontier and M. Trahin, Phys. Rev. 172, 83 (1968), Eqs. (1)-(13).

${ }^{8}$ Y. Gontier and M. Trahin, Phys. Rev. A 4, 1896 (1971).

${ }^{9}$ T. N. Chang and R. T. Poe, J. Phys. B 9, L311 (1976).

${ }^{10}$ M. Aymar and M. Crance, J. Phys. B 14, 3585 (1981).

${ }^{11}$ W. Zernik, Phys. Rev. 135, A51 (1964); W. Zernik and R. W. Klopfenstein, J. Math. Phys. 6, 262 (1965); W. Zernick,
} Phys. Rev. 176, 420 (1968).

${ }^{12}$ F. T. Chan and C. L. Tang, Phys. Rev. 185, 42 (1969).

13 S. Klarsfeld, Lett. Nuovo Cimento 2, 548 (1969).

${ }^{13 b}$ S. Klarsfeld, Lett. Nuovo Cimento 3, 395 (1970).

TABLE III. Three-photon ionization cross section $\left(\mathrm{cm}^{6} / \mathrm{W}^{2}\right)$ of $\mathrm{H}(1 s)$.

\begin{tabular}{cccccc}
\hline \hline & \multicolumn{2}{c}{ Linearly polarized light } & \multicolumn{2}{c}{ Circularly polarized light } \\
$\lambda(\AA)$ & LDFJR $^{\mathrm{a}}$ & $\mathrm{KV}^{\mathrm{b}}$ & Present & LDFJR $^{\mathrm{a}}$ & Present \\
\hline 1900 & $1.186 \times 10^{-46}$ & & $1.172 \times 10^{-46}$ & $2.479 \times 10^{-46}$ & $2.459 \times 10^{-46}$ \\
2000 & $5.581 \times 10^{-48}$ & $5.577 \times 10^{-48}$ & $5.429 \times 10^{-48}$ & $1.365 \times 10^{-47}$ & $1.326 \times 10^{-47}$ \\
2100 & $2.542 \times 10^{-47}$ & $2.582 \times 10^{-47}$ & $2.541 \times 10^{-47}$ & $5.771 \times 10^{-47}$ & $5.776 \times 10^{-47}$ \\
2200 & $1.593 \times 10^{-47}$ & $1.590 \times 10^{-47}$ & $1.589 \times 10^{-47}$ & $3.957 \times 10^{-47}$ & $3.945 \times 10^{-47}$ \\
2300 & $2.650 \times 10^{-47}$ & $2.599 \times 10^{-47}$ & $2.641 \times 10^{-47}$ & $3.853 \times 10^{-47}$ & $3.840 \times 10^{-47}$ \\
2400 & $7.125 \times 10^{-46}$ & $6.422 \times 10^{-46}$ & $7.057 \times 10^{-46}$ & $3.935 \times 10^{-47}$ & $3.917 \times 10^{-47}$ \\
2500 & $2.980 \times 10^{-46}$ & $3.034 \times 10^{-46}$ & $2.948 \times 10^{-46}$ & $3.998 \times 10^{-47}$ & $3.972 \times 10^{-47}$ \\
2600 & $1.008 \times 10^{-46}$ & $1.011 \times 10^{-46}$ & $1.002 \times 10^{-46}$ & $3.947 \times 10^{-47}$ & $3.906 \times 10^{-47}$ \\
\hline \hline
\end{tabular}


${ }^{14 a}$ E. Karule, J. Phys. B 4, L67 (1971).

${ }^{14 b}$ E. Karule, J. Phys. B 11, 441 (1978).

${ }^{15} \mathrm{G}$. Laplanche, A. Durrieu, Y. Flank, M. Jaouen, and A. Rachman, J. Phys. B 9, 1263 (1976).

${ }^{16}$ S. V. Khristenko and S. I. Vetchinkin, Opt. Spektrosk. 40, 417 (1976).

${ }^{17}$ A. Maquet, Phys. Rev. A 15, 1088 (1977).

${ }^{18}$ R. Shakeshaft, Phys. Rev. A 34, 5119 (1986).

${ }^{19}$ M. R. Teague and P. Lambropoulos, J. Phys. B 9, 1251 (1976).

${ }^{20}$ M. Edwards, X. Tang, P. Lambropoulos, and R. Shake- shaft, Phys. Rev. A 33, 4444 (1986).

${ }^{21}$ A. L'Huillier, L. Jönsson, and G. Wendin, Phys. Rev. A 33, 3938 (1986); A. L'Huillier and G. Wendin, J. Phys. B 20, L37 (1987).

${ }^{22}$ A. F. Starace and T. F. Jiang, Phys. Rev. A 36, 1705 (1987).

${ }^{23}$ Bo Gao and A. F. Starace, unpublished.

${ }^{24}$ Cf. Eq. (13) of Ref. 7.

${ }^{25}$ J. Nuttall and H. L. Cohen, Phys. Rev. 188, 1542 (1969). See also Ref. 5, Sec. VI.

${ }^{26}$ T. N. Rescigno, Phys. Rev. A 31, 607 (1985). 